\title{
Scientific outline of Claudio Zicovich-Wilson
}

\author{
Alejandro Ramírez-Solís ${ }^{1} \cdot$ Roberto Dovesi $^{2}$ (])
}

Published online: 10 July 2018

c) Springer-Verlag GmbH Germany, part of Springer Nature 2018

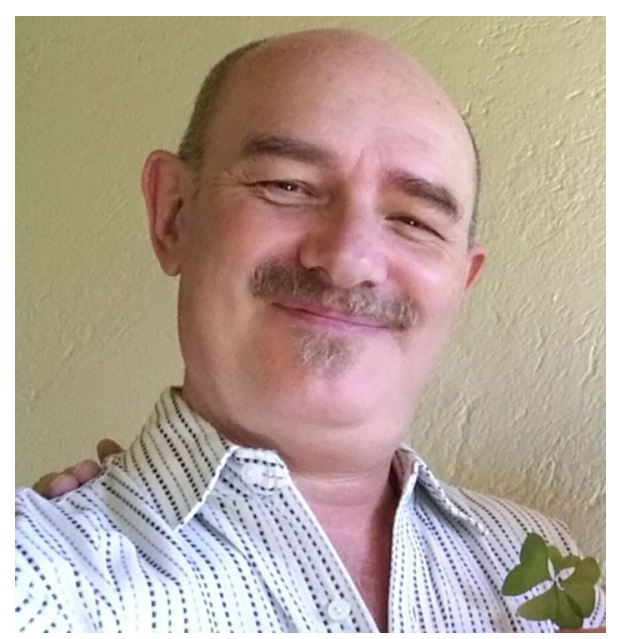

Claudio Marcelo Zicovich-Wilson was born on 20 October 1957, in full spring in Buenos Aires, Argentina. He was the second son of Bernardo and Sarita, the younger brother of Sergio Alejandro.

Claudio was always interested in chemistry. He was only 13 years old when, he and a close friend, mounted a chemistry laboratory in the washing room of the latter. They performed experiments with several compounds, metal oxides, bases, acids, Bunsen burners, using chemistry books as guides to their investigations.

Published as part of the special collection of articles "In Memoriam of Claudio Zicovich".

Alejandro Ramírez-Solís alex@uaem.mx

1 Centro de Investigación en Ciencias, Instituto de Investigación en Ciencias Básicas y Aplicadas, Universidad Autónoma del Estado de Morelos, 62209 Cuernavaca, Morelos, Mexico

2 Dipartimento di Chimica, Università degli studi di Torino, 10125 Turin, Italy
Claudio also developed a strong interest in music, and he learned to play the guitar. He was only 14 when started to compose and to play with some other friends.

In 1975 he enrolled in the Chemistry Program at the University of Buenos Aires. Those were very difficult times in Argentina due to the military dictatorship that ruled the country. Claudio was always ready to participate in musical or poetic events.

He was very interested and intensely studied linguistics, poetry, music, semiotics and pre-Socratic greek philosophy. He was particularly struck by the deep philosophical ideas of the I-Ching and the Tao-te-King. The discovery of Eastern philosophy, along with the practice of Qi Kung and Aikido, had deep implications for the rest of his life and his very generous stance toward fellow humans.

In mid-1979 Claudio left Argentina to spend a year visiting friends in Europe. He arrived in Madrid and then headed to Barcelona, where he met his wife-to-be, Lourdes. The first thing he did was to buy a good classical guitar from Valencia. He traveled through France, England, Italy and Holland, always going back to Barcelona between trips. He survived this period by delivering publicity and playing guitar in public places, metro stations or small cafés. He went back to Buenos Aires to finish his University studies by July 1980, with a longer hair, a good guitar and many more friends. He devoted his life to chemistry and music.

In 1982 Lourdes went to Buenos Aires and they got married in 1983. Their only son, Miguel, was born in 1984. In 1985 the family moved to Valencia, Spain.

From 1985 until 1994, with a family to feed, without a scholarship or stable income source, Claudio followed chemistry studies at the Universidad de Valencia, where he obtained his Ph.D. degree under the supervision of Avelino Corma and Pedro Viruela (thesis title: covalent interactions in reactions catalyzed by acidic zeolites). In 1994 he became a postdoctoral student working in catalysis problems at the Instituto de Tecnologías Químicas of the CSIC-Universidad Politécnica de Valencia (ITQ-UPV).

During that period, Claudio was part of two musical groups: AIMURAY, mainly playing andean music and a 
few own compositions, and later with the TENTENELAIRE group, playing many more original fusion compositions. He was also a founder member of the pottery workshop ARTANA.

From 1995 to 1997 he was a postdoctoral fellow at the Chemistry Department, Universitá di Torino, Italy. Roberto Dovesi met him in 1994, when he was invited by Avelino Corma to visit his group in Valencia. The main reason for the visit was Claudio's interest to be involved in a more fundamental theoretical work, as in Valencia his role was essentially to support the experimental activity of the group, one of the world leader Institutions in catalysis.

When Claudio arrived in Torino, Roberto proposed him a series of possible research investigations, spanning from formalisms the Torino group was trying to implement in CRYSTAL, to applications in catalysis, along the lines of his previous activity. On top of the list, in terms of difficulty, was the implementation of the SACO (Symmetry Adapted Crystalline Orbitals) representation in the CRYSTAL code. This was an idea that Roberto had in mind for a long time, but that had never been implemented in periodic codes. At that time, and for many years since then, the equivalent SAMO (Symmetry Adapted Molecular Orbitals) were implemented in molecular codes just for abelian groups (with no degeneracies). The advantages of SACO's are evident: the Fock matrix to be diagonalized becomes block diagonal according to the various Irreducible Representations (IR) of the group and the factorization extends also to the different rows of the IR.

He accepted immediately the most challenging task. Roberto was worried about the difficulties, as the documentation on the matter was scarce and very "mathematical." So he was not sure that they (actually Claudio) could get at the end of the project: every day Roberto was entering in his office, trying to discuss with him the various aspects, and implicitly trying to understand if he was solid enough to win this very hard battle. But after a week, Claudio asked Roberto to leave him alone with the problem: "please, do no ask me; when the problem will be solved, I will inform you and provide you the solution." And this is what happened: after 6 months he found the way to solve the problem of generating the SACOs for any Space Group, without any additional information other than the operators of the group. This leads to two publications $[115,116]$ and to the implementation of SACOs in the CRYSTAL code that remains a unique, not only in periodic codes, but also in the molecular context, as documented by two papers published about 16 years later $[22,23]$, showing that, using SACO's, it has been possible to investigate fullerenes much larger than the ones previously investigated with molecular codes: icosahedral fullerenes up to $(10,10)$, that contain 6000 atoms; in spite of the dimension of the basis set ( 84.000 functions), the full SCF has been performed in $24 \mathrm{~h}$ on a single core; the reason being that the size of the largest matrix to be diagonalized drops down to 3600 and that along the calculation all linear algebra is performed in the SACO basis rather than in the AO basis (in this last part of the work, Roberto Orlando and Marco De La Pierre have been involved).

From these years, and until his sudden and unexpected death, Claudio became a constant and very bright collaborator of the CRYSTAL project. Fully devoted to the project, he was visiting Torino at least once a year (for a month or more) attending to most of the CRYSTAL schools, helping Ph.D. and undergraduate students in solving problems, being very precious in debugging the code.

During the last 20 years, he contributed to many other new features of the CRYSTAL code. In 1996 he visited Daresbury, and with Vic Saunders (another of the major contributors to the code) he implemented a scheme for the generation of Wannier functions from the Crystalline Orbitals in the basis of the Bloch functions. The scheme was published only 4 years later [107], because both Claudio and Vic were very often trapped solving "the next" problem, and not very attracted by the idea of describing what was already working properly. From 2004, starting from the analytical gradient implemented by Klaus Doll in Daresbury under the supervision of Vic Saunders and a crude version of the optimizer for both cartesian coordinates and unit cell parameters, proposed by Philippe D'Arco, Claudio implemented a solid, versatile, robust, accurate tool for the optimization of structures, that remains effective also when applied to very large unit cell systems, as it has been the case of bulk Crambin, or huge fullerenes [23]. The list of Claudio's crucial contributions could continue, including the IR intensities evaluated from the Wannier functions, a sophisticated scheme for the determination of Transition States [47], and a scheme for the evaluation the Hirshfeld charges for periodic systems [11]. Claudio (with Fabien Pascale) implemented most of the routines for the calculation of the vibrational frequencies [95], and many options related to their analysis, including the correspondence between LO and TO modes, the automatic symmetry classification of the modes on the basis of the IR of the space group. Claudio has been one of the main authors of 5 major releases of the program (CRYSTAL98, 03, 06, 09 and 14) and a lecturer to most of the CRYSTAL-based summer schools that have been held in the last 20 years in Italy, Spain, USA, Germany, UK, France, México, Argentina, Cuba and Brazil. He authored several book chapters and 136 papers published in international journals with over 9500 citations.

He spent several short research periods in many places, such as the Universidad Jaume I in Castellón (Spain), Dirección de Cómputo Académico at the Universidad Nacional Autónoma de México, Laboratoire de Physique Quantique (LPQ) at the Université de Toulouse, le Departement de Géologie de l'École Nationale Supérieure in Paris, the 
Institut Charles Gerhardt, Montpellier, and the Computational Science Department at CCLRC- Daresbury Laboratory in the UK. The themes he worked on ranged from extremely theoretical (the development of a Difference-Dedicated Configuration Interaction scheme for a multiconfigurational reference wavefunction in LPQ in Toulouse under Jean-Pierre Daudey) to specifically applied practical problems (the study of molecular interactions in crucial geologic compounds and volcanic chemical reactions) in ENS-Paris and UNAM-México.

In 1998 he went back to Valencia and worked in catalytic materials and reactions as Associate Researcher at the ITQUPV until 2001, when, invited by Alejandro Ramírez-Solís, he took a full Professor position at the Physics Department, Facultad de Ciencias, Universidad Autónoma del Estado de Morelos in Cuernavaca, México. In the last decade, he tackled with Alejandro fundamental problems in metaldoped organic polymers and the complex electronic structure problem posed by the high-pressure $\varepsilon$ and $\zeta$ phases of solid oxygen; in the latter case, they were able to show that the previous failures of GGA-DFT to deal with structural, vibrational and energetic properties were due to the rather strong multi-reference character of the $\left(\mathrm{O}_{2}\right)_{4}$ unit cell of these phases and that only hybrid DFT methods can accurately describe them. He stayed as full Professor there until his untimely departure.

Because of his human and empathic character, he has never just been a colleague or collaborator but rather a caring son, brother, uncle, father and friend to many of us during all these years.

He was fundamentally gentle and kind to everyone he met. We will all miss his irony, his Argentinian humor, his colorful shirts and tasteful hats, and the beautiful sound of his guitar.

\section{List of scientific publications}

1. A Albavera-Mata, CM Zicovich-Wilson, JL Gázquez, SB Trickey, A Vela. Long-Range Exchange Limit and Dispersion in Pure Silica Zeolites. Theor Chem. Acc. 2018, 137 (2), 26.

2. R Dovesi, A Erba, R Orlando, CM Zicovich-Wilson, B Civalleri, L Maschio, M Rérat, S Casassa, J Baima, S Salustro, B Kirtman. Quantum-Mechanical Condensed Matter Simulations with Crystal. Wiley Interdiscip Rev Comput Mol Sci. 2018, https://doi.org/10.1002/ wcms.1360.

3. S Salustro, Y Nöel, CM Zicovich-Wilson, P Olivero, R Dovesi. The V + I Defects in Diamond: An Ab Initio Investigation of the Electronic Structure, of the Raman and IR Spectra, and of Their Possible Recombination.
J. Chem. Phys. 2016, 145 (18), 184701. https://doi. org/10.1063/1.4966635.

4. S Salustro, G Sansone, CM Zicovich-Wilson, Y Noël, L Maschio, R Dovesi. The A-Center Defect in Diamond: Quantum Mechanical Characterization through the Infrared Spectrum. Phys. Chem. Chem. Phys. 2017, 19 (22), 14478-14485. https://doi.org/10.1039/c7cp0 $0093 \mathrm{f}$.

5. K Valdiviés-Cruz, A Lam, CM Zicovich-Wilson. Full Mechanism of Zeolite Dealumination in Aqueous Strong Acid Medium: Ab Initio Periodic Study on H-Clinoptilolite. J. Phys. Chem. C 2017, 121 (5), 2652-2660. https://doi.org/10.1021/acs.jpcc.6b09794.

6. A Erba, D Caglioti, CM Zicovich-Wilson, R Dovesi. Nuclear-relaxed Elastic and Piezoelectric Constants of Materials: Computational Aspects of Two Quantummechanical Approaches. J. Comput. Chem. 2017, 38 (5), 257-264. https://doi.org/10.1002/jcc.24687.

7. A Ramírez-Solís A, CM Zicovich-Wilson, R Hernández-Lamoneda, AJ Ochoa-Calle. Antiferromagnetic vs. Non-Magnetic $\varepsilon$ Phase of Solid Oxygen. Periodic Density Functional Theory Studies Using a Localized Atomic Basis Set and the Role of Exact Exchange. Phys. Chem. Chem. Phys. 2017, 19 (4), 2826-2833. https://doi.org/10.1039/c6cp07445f.

8. S Salustro, A Erba, CM Zicovich-Wilson, Y Nöel, L Maschio, R Dovesi. Infrared and Raman Spectroscopic Features of the Self-Interstitial Defect in Diamond from Exact-Exchange Hybrid DFT Calculations. Phys. Chem. Chem. Phys. 2016, 18 (31), 21288-21295. https ://doi.org/10.1039/c6cp02403c.

9. R Demichelis, M De La Pierre, M Mookherjee, CM Zicovich-Wilson, R Orlando. Serpentine Polymorphism: A Quantitative Insight from First-Principles Calculations. CrystEngComm 2016, 18 (23), 44124419 https://doi.org/10.1039/c6ce00190d.

10. AM Navarrete-López, ML San-Román, CM ZicovichWilson. The Influence of the DFT Approach on the Structure and Relative Stability of Models for Cellulose I Allomorphs. Theor. Chem. Acc. 2016, 135 (5), 136. https://doi.org/10.1007/s00214-016-1889-6.

11. CM Zicovich-Wilson, M Hô, AM Navarrete-López, S. Casassa. Hirshfeld-I Charges in Linear Combination of Atomic Orbitals Periodic Calculations. Theor. Chem. Acc. 2016, 135(18), 188. https://doi.org/10.1007/s0021 4-016-1942-5.

12. J Baima, A Erba, L Maschio, CM Zicovich-Wilson, R Dovesi, B Kirtman. Direct Piezoelectric Tensor of 3D Periodic Systems Through a Coupled Perturbed Hartree-Fock/Kohn-Sham Method. Zeitschrift für Phys. Chemie 2016, 230 (5-7), 719-736. https://doi. org/10.1515/zpch-2015-0701. 
13. K Valdiviés-Cruz, A Lam, CM Zicovich-Wilson. Chemical Interaction of Water Molecules with Framework Al in Acid Zeolites: A Periodic Ab Initio Study on H-Clinoptilolite. Phys. Chem. Chem. Phys. 2015, 17(36), 23657-23666. https://doi.org/10.1039/c5cp0 $3268 \mathrm{~g}$.

14. AJ Ochoa-Calle, CM Zicovich-Wilson, R Hernández, A Ramírez-Solís. Understanding the $\varepsilon$ and $\zeta$ HighPressure Solid Phases of Oxygen. Systematic Periodic Density Functional Theory Studies Using Localized Atomic Basis. J. Chem. Theory Comput. 2015, 11 (3), 1195-1205. https://doi.org/10.1021/acs.jctc.5b00017.

15. A Erba, AM Navarrete-López, V Lacivita, P D’Arco, CM Zicovich-Wilson. Katoite under Pressure: An $\mathrm{Ab}$ Initio Investigation of Its Structural, Elastic and Vibrational Properties Sheds Light on the Phase Transition. Phys. Chem. Chem. Phys. 2015, 17 (4), 26602669. https://doi.org/10.1039/c4cp04414b.

16. EI Román-Román, CM Zicovich-Wilson. The Role of Long-Range van Der Waals Forces in the Relative Stability of $\mathrm{SiO}_{2}$-Zeolites. Chem. Phys. Lett. 2015, 619, 109-114. https://doi.org/10.1016/j.cplett.2014.11.044.

17. AJ Ochoa-Calle, CM Zicovich-Wilson, A RamírezSolís. Solid Oxygen $\zeta$ Phase and its Transition from the $\varepsilon$ Phase at Extremely High Pressure: A First-Principles Analysis. Phys. Rev. B 2015, 92 (8), 085148. https:// doi.org/10.1103/physrevb.92.085148.

18. AJ Ochoa-Calle, CM Zicovich-Wilson, A RamírezSolís. On the Raman and Infrared Vibrational Spectra of the $\varepsilon$ and $\zeta$ Phases of Solid Oxygen. Systematic DFT Studies with Localized Basis Sets. Chem. Phys. Lett. 2015, 638, 82-86. https://doi.org/10.1016/j.cplet t.2015.08.036.

19. M De La Pierre, R Orlando, M Ferrabone, CM Zicovich-Wilson, R Dovesi. Exploitation of Symmetry in Periodic Self-Consistent-Field Ab Initio Calculations: Application to Large Three-Dimensional Compounds. Sci. China Chem. 2014, 57 (10), 1418-1426. https:// doi.org/10.1007/s11426-014-5191-y.

20. J Shin, N Ho-Ahn, MA Camblor, CM Zicovich-Wilson, SB Hong. Synthesis of Aluminosilicate Natrolites and Control of Their Tetrahedral Atom Ordering. Chem. Mater. 2014, 26 (11), 3361-3363. https://doi. org/10.1021/cm5013763.

21. K Valdiviés-Cruz, A Lam, CM Zicovich-Wilson. Periodic Quantum Chemical Studies on Anhydrous and Hydrated Acid Clinoptilolite. J. Phys. Chem. A 2014, 118 (31), 5779-5789. https://doi.org/10.1021/jp410 $754 \mathrm{a}$.

22. R Orlando, M De La Pierre, CM Zicovich-Wilson, A Erba, R Dovesi. On the Full Exploitation of Symmetry in Periodic (as Well as Molecular) Self-Consist-
ent-Field Ab Initio Calculations. J. Chem. Phys. 2014, 141(10), 104108. https://doi.org/10.1063/1.4895113.

23. Y Noël, M De La Pierre, CM Zicovich-Wilson, R Orlando, R Dovesi. Structural, Electronic and Energetic Properties of Giant Icosahedral Fullerenes up to $\mathrm{C}_{6000}$ : Insights from an Ab Initio Hybrid DFT Study. Phys. Chem. Chem. Phys. 2014, 16 (26), 13390-13401. https://doi.org/10.1039/c4cp01442a.

24. R Dovesi, R Orlando, A Erba, CM Zicovich-Wilson, B Civalleri, S Casassa, L Maschio, M Ferrabone, M De La Pierre, Ph D’Arco, Y Noël, M Causà, M Rérat, B. Kirtman. CRYSTAL14: A Program for the Ab Initio Investigation of Crystalline Solids. Int. J. Quantum Chem. 2014, 114 (19), 1287-1317. https://doi. org/10.1002/qua.24658.

25. A Ramírez-Solís, R Bernal-Jaquez, CM Zicovich-Wilson. The Molecular and Electronic Structure of Poly[2,7-(Benzo [2,1; 3,4-B'] Dithiophene)-Alt-2, 2'-(3, 3'-Didodecyl-5, 5'-bithiophenyl)](PBTT): A Periodic DFT Approach. Chem. Phys. Lett. 2014, 607, 47-51. https://doi.org/10.1016/j.cplett.2014.05.047.

26. KE El-Kelany, M Ferrabone, M Rérat, Ph Carbonnière, CM Zicovich-Wilson, R Dovesi. The Electronic Structure of $\mathrm{MgO}$ Nanotubes. An Ab Initio Quantum Mechanical Investigation. Phys. Chem. Chem. Phys. 2013, 15 (32), 13296-13303 https://doi.org/10.1039/ c3cp50979f.

27. S Sene, M Reinholdt, G Renaudin, D Berthomieu, CM Zicovich-Wilson, CG.; Gaveau, P.; Bonhomme, C.; Filinchuk, Y.; Smith, M. E.; Nedelec, J.-M.; Bégu, S.; Mutin, P. Hubert, D Laurencin. Boronate Ligands in Materials: Determining Their Local Environment by Using a Combination of IR/Solid-State NMR Spectroscopies and DFT Calculations. Chem. Eur. J. 2013, 19 (3), 880-891 https://doi.org/10.1002/chem.201203560.

28. A Rojas, ML San-Roman, CM Zicovich-Wilson, MA Camblor. Host-Guest Stabilization of a Zeolite Strained Framework: In Situ Transformation of Zeolite MTW into the Less Dense and More Strained ITW. Chem. Mater. 2013, 25 (5), 729-738. https://doi. org/10.1021/cm303709e.

29. L Maschio, B Kirtman, S Salustro, CM ZicovichWilson, R Orlando, R Dovesi. Raman Spectrum of Pyrope Garnet. A Quantum Mechanical Simulation of Frequencies, Intensities, and Isotope Shifts. J. Phys. Chem. A 2013, 117 (45), 11464-11471. https://doi. org/10.1021/jp4099446.

30. M Ho, AM Navarrete-López, CM Zicovich-Wilson, A Ramírez-Solís. Electronic Charge Density Analysis of Li-Doped Polyacetylene: Molecular vs. Periodic Descriptions and Nature of Li-to-Chain Bonding. $J$. Phys. Chem. B 2013, 117 (2), 725-730. https://doi. org/10.1021/jp3106343. 
31. CM Zicovich-Wilson, Y Noel, AM Ferrari, R Orlando, $\mathrm{M}$ De La Pierre, R Dovesi. On the Use of Symmetry in SCF Calculations. The Case of Fullerenes and Nanotubes. AIP Conf. Proc. 2012, 1456 (1), 248-255. https ://doi.org/10.1063/1.4730666.

32. CM Zicovich-Wilson, A Erba. Beyond Wigner's Theorems: The Role of Symmetry Equivalences in Quantum Systems. Int. J. Quantum Chem. 2012, 112 (21), 3543-3551. https://doi.org/10.1002/qua.24184.

33. A Meyer, M Ferrero, L Valenzano, CM Zicovich-Wilson, R Orlando, R Dovesi. Coupled Perturbed HF/KS Calculation of the Dielectric Constant of Crystalline Systems. The Case of Six Members of the Garnet Family. AIP Conf. Proc. 2012, 1504 (1), 593-596. https:// doi.org/10.1063/1.4771766.

34. Y Noël, M de la Pierre, L Maschio, M Rérat, CM Zicovich-Wilson, R Dovesi. Electronic Structure, Dielectric Properties and Infrared Vibrational Spectrum of Fayalite: An Ab Initio Simulation with an All-Electron Gaussian Basis Set and the B3LYP Functional. Int. J. Quantum Chem. 2012, 112 (9), 2098-2108. https://doi. org/10.1002/qua.23195.

35. A Rojas, E Martínez-Morales, CM Zicovich-Wilson. Zeolite Synthesis in Fluoride Media: Structure Direction toward ITW by Small Methylimidazolium Cations. J. Am. Chem. Soc. 2012, 134 (4), 2255-2263. https://doi.org/10.1021/ja209832y.

36. JC Tan, B Civalleri, CC Lin, L Valenzano, R Galvelis, PF Chen, TD Bennett, C Mellot-Draznieks, CM Zicovich-Wilson, AK Cheetham. Exceptionally Low Shear Modulus in a Prototypical Imidazole-Based MetalOrganic Framework. Phys. Rev. Lett. 2012, 108 (9), 095502. https://doi.org/10.1103/physrevlett.108.09550 2.

37. E Martínez-Morales, CM Zicovich-Wilson. Adjusting Framework Ionicity to Favour Crystallisation of Zeolites with Strained Structural Units. Periodic Quantum Chemical Studies. Catal. Sci. Technol. 2011, 1 (6), 868-878. https://doi.org/10.1039/c1cy00010a.

38. R Colle, G Grosso, A Ronzani, CM Zicovich-Wilson. Structure and X-ray Spectrum of Crystalline Poly (3-hexylthiophene) from DFT-van der Waals Calculations. Phys. status solidi 2011, 248 (6), 1360-1368. https://doi.org/10.1002/pssb.201046429.

39. R Demichelis, Y Noel, Ph D’Arco, M Rérat, CM Zicovich-Wilson, R Dovesi. Properties of Carbon Nanotubes: An Ab Initio Study Using Large Gaussian Basis Sets and Various DFT Functionals. J. Phys. Chem. C 2011, 115 (18), 8876-8885. https://doi. org/10.1021/jp110704x.

40. R Demichelis, Y Noël, P Ugliengo, CM ZicovichWilson, R Dovesi. Physico-Chemical Features of Aluminum Hydroxides as Modeled with the Hybrid
B3LYP Functional and Localized Basis Functions. $J$. Phys. Chem. C 2011, 115 (27), 13107-13134. https:// doi.org/10.1021/jp200523x.

41. GF Catá, HC Rojas, AP Gramatges, CM ZicovichWilson, LJ Álvarez, C Searle. Initial Structure of Cetyltrimethylammonium Bromide Micelles in Aqueous Solution from Molecular Dynamics Simulations. Soft Matter 2011, 7 (18), 8508-8515. https://doi. org/10.1039/c1sm05127j.

42. R Dovesi, M De La Pierre, AM Ferrari, F Pascale, L Maschio, CM Zicovich-Wilson. The IR Vibrational Properties of Six Members of the Garnet Family: A Quantum Mechanical Ab Initio Study. Am. Mineral. 2011, 96 (11-12), 1787-1798. https://doi.org/10.2138/ am.2011.3804.

43. CM Zicovich-Wilson, A Erba. A Fundamental Connection between Symmetry and Spatial Localization Properties of Basis Sets. Theor Chem Acc 2010, 126 (3-4), 165-175. https://doi.org/10.1007/s00214-0090619-8.

44. J Graciani, AM Márquez, JJ Plata, Y Ortega, NC Hernández, A Meyer, CM Zicovich-Wilson, J Fernández-Sanz. Comparative Study on the Performance of Hybrid DFT Functionals in Highly Correlated Oxides: The Case of $\mathrm{CeO}_{2}$ and $\mathrm{Ce}_{2} \mathrm{O}_{3}$. J. Chem. Theory Comput. 2010, 7 (1), 56-65. https://doi.org/10.1021/ct100 430q.

45. Y Noel, Ph D’arco, R Demichelis, CM Zicovich-Wilson, R Dovesi. On the Use of Symmetry in the Ab Initio Quantum Mechanical Simulation of Nanotubes and Related Materials. J. Comput. Chem. 2010, 31 (4), 855-862. https://doi.org/10.1002/jcc.21370.

46. B Civalleri, L Maschio, P Ugliengo, CM ZicovichWilson. Role of Dispersive Interactions in the $\mathrm{CO}$ Adsorption on $\mathrm{MgO}$ (001): Periodic B3LYP Calculations Augmented with an Empirical Dispersion Term. Phys. Chem. Chem. Phys. 2010, 12 (24), 6382-6386. https://doi.org/10.1039/c001192d.

47. A Rimola, CM Zicovich-Wilson, R Dovesi, $\mathrm{P}$ Ugliengo. Search and Characterization of Transition State Structures in Crystalline Systems Using Valence Coordinates. J. Chem. Theory Comput. 2010, 6 (4), 1341-1350. https://doi.org/10.1021/ct900680f.

48. A Meyer, F Pascale, CM Zicovich-Wilson, R Dovesi. Magnetic Interactions and Electronic Structure of Uvarovite and Andradite Garnets. An Ab Initio AllElectron Simulation with the CRYSTAL06 Program. Int. J. Quantum Chem. 2010, 110 (2), 338-351. https ://doi.org/10.1002/qua.22302.

49. CM Zicovich-Wilson, F Gándara, A Monge, MA Camblor. In Situ Transformation of TON Silica Zeolite into the Less Dense ITW: Structure-Direction Overcoming Framework Instability in the Synthesis of SiO2 Zeo- 
lites. J. Am. Chem. Soc. 2010, 132 (10), 3461-3471. https://doi.org/10.1021/ja9094318.

50. L Gómez-Hortigüela, F Corà, G Sankar, CM ZicovichWilson, CRA Catlow. Catalytic Reaction Mechanism of Mn-Doped Nanoporous Aluminophosphates for the Aerobic Oxidation of Hydrocarbons. Chem. Eur. J. 2010, 16 (46), 13638-13645. https://doi.org/10.1002/ chem. 201001876.

51. AM Ferrari, D Szieberth, CM Zicovich-Wilson, R Dovesi. Anatase(001) 3 ML Nanotubes, The First TiO2 Nanotube With Negative Strain Energies: A DFT Prediction. J. Phys. Chem. Lett. 2010, 1 (19), 2854-2857. https://doi.org/10.1021/jz101184f.

52. AM Walker, B Civalleri, B Slater, C Mellot-Draznieks, F Corá, CM Zicovich-Wilson, G Roman-Pérez, JM Soler, JD Gale. Flexibility in a Metal—Organic Framework Material Controlled by Weak Dispersion Forces : The Bistability of MIL-53 (Al)*. Angew. Chemie Int. Ed. 2010, 49 (41), 7501-7503. https://doi.org/10.1002/ anie.201002413.

53. CM Zicovich-Wilson, B Kirtman, B Civalleri, A Ramírez-Solís. Periodic Density Functional Theory Calculations for 3-Dimensional Polyacetylene with Empirical Dispersion Terms. Phys. Chem. Chem. Phys. 2010, 12 (13), 3289-3293 https://doi.org/10.1039/ b918539a.

54. CM Zicovich-Wilson, ML San Román, A RamírezSolís. Mechanism of F-Elimination from Zeolitic D4R Units: A Periodic B3LYP Study on the Octadecasil Zeolite. J. Phys. Chem. C 2010, 114 (7), 2989-2995. https://doi.org/10.1021/jp9088244.

55. A Rimola, M Corno, CM Zicovich-Wilson, $\mathrm{P}$ Ugliengo. Ab Initio Modeling of Protein/biomaterial Interactions: Competitive Adsorption between Glycine and Water onto Hydroxyapatite Surfaces. Phys. Chem. Chem. Phys. 2009, 11 (40), 9005-9007. https://doi. org/10.1039/b913311a.

56. G Fernández-Catá, A Pérez-Gramatges, LJ Alvarez, H Comas-Rojas, CM Zicovich-Wilson. On the Interaction between Silica Surfaces and Surfactants. A 2D Periodic B3LYP Investigation. J. Phys. Chem. C 2009, 113 (30), 13309-13316. https://doi.org/10.1021/jp901 $320 \mathrm{q}$.

57. B Civalleri, P Ugliengo, CM Zicovich-Wilson, R Dovesi. Ab Initio Modeling of Layered Materials with the CRYSTAL Code: An Overview. Zeitschrift für Krist.: Cryst. Mater. 2009, 224 (5-6), 241-250. https ://doi.org/10.1524/zkri.2009.1144.

58. R Credendino, V Busico, M Causà, V Barone, PHM Budzelaar, CM Zicovich-Wilson. Periodic DFT Modeling of Bulk and Surface Properties of $\mathrm{MgCl}$ 2. Phys. Chem. Chem. Phys. 2009, 11 (30), 6525-6532. https ://doi.org/10.1039/b905676a.
59. A Ramírez-Solís, B Kirtman, R Bernal-Jáquez, CM Zicovich-Wilson. Periodic Density Functional Theory Studies of Li-Doped Polythiophene: Dependence of Electronic and Structural Properties on Dopant Concentration. J. Chem. Phys. 2009, 130 (16), 164904. https://doi.org/10.1063/1.3109079.

60. R Dovesi, L Valenzano, F Pascale, CM ZicovichWilson, R Orlando. Ab Initio Quantum-Mechanical Simulation of the Raman Spectrum of Grossular. $J$. Raman Spectrosc. 2009, 40 (4), 416-418. https://doi. org/10.1002/jrs.2144.

61. P Ugliengo, CM Zicovich-Wilson, S Tosoni, B. C. Role of Dispersive Interactions in Layered Materials: A Periodic B3LYP and B3LYP-D* Study of $\mathrm{Mg}(\mathrm{OH})$ 2, $\mathrm{Ca}(\mathrm{OH}) 2$ and Kaolinite. J. Mater. Chem. 2009, 19 (17), 2564-2572 https://doi.org/10.1039/b819020h.

62. CM Zicovich-Wilson. Two Points of View to Look at Symmetry. J. Phys. Conf. Ser. 2008, 117, 012030. https ://doi.org/10.1088/1742-6596/117/1/012030.

63. R Demichelis, Y Noel, CM Zicovich-Wilson, C Roetti, L Valenzano, R Dovesi. Ab-Initio Quantum Mechanical Study of Akdalaite (5Al2O3centerdot H2O): Structure and Vibrational Spectrum. J. Phys. Conf. Ser. 2008, 117 (1), 012013. https://doi.org/10.1088/17426596/117/1/012013.

64. CM Zicovich-Wilson, FJ Torres, F Pascale, L Valenzano, R Orlando, R Dovesi. Ab Initio Simulation of the IR Spectra of Pyrope, Grossular, and Andradite. J. Comput. Chem. 2008, 29 (13), 2268-2278. https://doi. org/10.1002/jcc.20993.

65. B Civalleri, CM Zicovich-Wilson, L Valenzano, P Ugliengo. B3LYP Augmented with an Empirical Dispersion Term (B3LYP-D*) as Applied to Molecular Crystals. CrystEngComm 2008, 10 (4), 405-410 https ://doi.org/10.1039/b715018k.

66. A Ramírez-Solís, B Kirtman, R Bernal-Jáquez, CM Zicovich-Wilson. Periodic Density Functional Theory Calculations for Na-Doped Quasi-One-Dimensional Polyacetylene Chains. J. Phys. Chem. C 2008, 112 (25), 9493-9500. https://doi.org/10.1021/jp0774261.

67. B Civalleri, K Doll, CM Zicovich-Wilson. Ab Initio Investigation of Structure and Cohesive Energy of Crystalline Urea. J. Phys. Chem. B 2007, 111 (1), 26-33. https://doi.org/10.1021/jp065757c.

68. CM Zicovich-Wilson, ML San-Román, MA Camblor, F Pascale, J. S. D.-N. Structure, Vibrational Analysis, and Insights into Host-Guest Interactions in AsSynthesized Pure Silica ITQ-12 Zeolite by Periodic B3LYP Calculations. J. Am. Chem. Soc. 2007, 129 (37), 11512-11523. https://doi.org/10.1021/ja073 0361.

69. AR Albunia, P Rizzo, G Guerra, FJ Torres, B Civalleri, CM Zicovich-Wilson. Uniplanar Orientations as 
a Tool to Assign Vibrational Modes of Polymer Chain. Macromolecules 2007, 40 (11), 3895-3897. https://doi. org/10.1021/ma070380+.

70. L Valenzano, Y Noel, R Orlando, CM Zicovich-Wilson, M Ferrero, R Dovesi. Ab Initio Vibrational Spectra and Dielectric Properties of Carbonates: Magnesite, Calcite and Dolomite. Theor. Chem. Accounts Theory, Comput. Model. (Theoretica Chim. Acta) 2007, 117 (5-6), 991-1000. https://doi.org/10.1007/s00214-0060213-2.

71. G Ottonello, B Civalleri, M Vetuschi Zuccolini, CM Zicovich-Wilson. Ab-Initio Thermal Physics and Cr-Isotopic Fractionation of $\mathrm{MgCr} 2 \mathrm{O} 4$. Am. Mineral. 2007, 92 (1), 98-108. https://doi.org/10.2138/ am.2007.2213.

72. G Herrera-Pérez, CM Zicovich-Wilson, A RamírezSolís. Periodic DFT Studies of AlPO-11: The Role of Hydration on Structural Properties. J. Phys. Chem. C 2007, 111 (27), 9664-9670. https://doi.org/10.1021/ jp072004x.

73. N Cruz Hernandez, CM Zicovich-Wilson, J Fernández-Sanz. The Constrained Space Orbital Variation Analysis for Periodic Ab Initio Calculations. J. Chem. Phys. 2006, 124 (19), 194105. https://doi. org/10.1063/1.2198528.

74. S Casassa, CM Zicovich-Wilson, C. P. SymmetryAdapted Localized Wannier Functions Suitable for Periodic Local Correlation Methods. Theor. Chem. Accounts Theory, Comput. Model. 2006, 116 (4-5), 726-733. https://doi.org/10.1007/s00214-006-0119-z.

75. R Orlando, FJ Torres, F Pascale, P Ugliengo, CM Zicovich-Wilson, R Dovesi. Vibrational Spectrum of Katoite Ca3Al2 [(OH) 4] 3: A Periodic Ab Initio Study. J. Phys. Chem. B 2006, 110 (2), 692-701. https ://doi.org/10.1021/jp053602j.

76. B Montanari, B Civalleri, CM Zicovich-Wilson, R Dovesi. Influence of the Exchange-correlation Functional in All-Electron Calculations of the Vibrational Frequencies of Corundum $\left(\alpha-\mathrm{Al}_{2} \mathrm{O}_{3}\right)$. Int. J. Quantum Chem. 2006, 106 (7), 1703-1714. https://doi. org/10.1002/qua.20938.

77. J Scaranto, G Mallia, S Giorgianni, CM Zicovich-Wilson, B Civalleri, N Harrison. A Quantum-Mechanical Study of the Vinyl Fluoride Adsorbed on the Rutile $\mathrm{TiO}_{2}(1110)$ Surface. Surf. Sci. 2006, 600 (2), 305-317. https://doi.org/10.1016/j.susc.2005.10.032.

78. L Valenzano, FJ Torres, K Doll, F Pascale, CM Zicovich-Wilson, R Dovesi. Ab Initio Study of the Vibrational Spectrum and Related Properties of Crystalline Compounds; the Case of $\mathrm{CaCO}_{3}$ Calcite. Zeitschrift für Phys. Chemie 2006, 220 (7/2006), 893-912. https ://doi.org/10.1524/zpch.2006.220.7.893.
79. A Ramírez-Solís, CM Zicovich-Wilson, B Kirtman. Periodic Hartree-Fock and Density Functional Theory Calculations for Li-Doped Polyacetylene Chains. J. Chem. Phys. 2006, 124 (24), 244703. https://doi. org/10.1063/1.2208363.

80. G Fernández-Catá, LJ Álvarez, R Dovesi, C. ZicovichWilson. Back-Donation in the Electronic Structure of AlN(W) Surfaces. A Periodic ab initio Study. Rev. Cuba. Química 2005, 17 (2), 170. http://www.redal yc.org/pdf/4435/443543686036.pdf.

81. P Ugliengo, C Busco, B Civalleri, CM ZicovichWilson. Carbon Monoxide Adsorption on Alkali and Proton-Exchanged Chabazite: An Ab-Initio Periodic Study Using the CRYSTAL Code. Mol. Phys. 2005, 103 (18), 2559-2571. https://doi.org/10.1080/00268 970500180865.

82. P Labeguerie, F Pascale, M Merawa, CM ZicovichWilson, N Makhouki, R Dovesi. Phonon Vibrational Frequencies and Elastic Properties of Solid $\mathrm{SrFCl}$. An Ab Initio Study. Eur. Phys. J. B-Condensed Matter Complex Syst. 2005, 43 (4), 453-461. https://doi. org/10.1140/epjb/e2005-00078-6.

83. F Pascale, CM Zicovich-Wilson, R Orlando, C Roetti, P Ugliengo, R Dovesi. Vibration Frequencies of Mg3Al2Si3O12 Pyrope. An Ab Initio Study with the CRYSTAL Code. J. Phys. Chem. B 2005, 109 (13), 6146-6152. https://doi.org/10.1021/jp050316z.

84. C Pisani, M Busso, G Capecchi, S Casassa, R Dovesi, L Maschio, CM Zicovich-Wilson, M Schütz. LocalMP2 Electron Correlation Method for Nonconducting Crystals. J. Chem. Phys. 2005, 122 (9), 094113. https ://doi.org/10.1063/1.1857479.

85. R Dovesi, R Orlando, B Civalleri, C Roetti, VR Saunders, CM Zicovich-Wilson. CRYSTAL: A Computational Tool for the Ab Initio Study of the Electronic Properties of Crystals. Zeitschrift für Krist. Mater. 2005, 220 (5/6), 571-573 https://doi.org/10.1524/ zkri.220.5.571.65065.

86. PM Esteves, CL Araújo, BAC Horta, LJ Alvarez, CM Zicovich-Wilson, A Ramírez-Solís. The IsobutyleneIsobutane Alkylation Process in Liquid HF Revisited. J. Phys. Chem. B 2005, 109 (26), 12946-12955. https ://doi.org/10.1021/jp051567a.

87. G Fernández-Catá, LJ Álvarez, R Dovesi, CM Zicovich-Wilson. Periodic Ab Initio Study of the Electronic Structure of $\alpha-\mathrm{Al} 2 \mathrm{O} 3$ and AlN (W) Surfaces Based on Localized Wannier Functions. J. Phys. Chem. B 2004, 108 (22), 7316-7322. https://doi.org/10.1021/jp037 1736.

88. SB Hong, SH Lee, CH Shin, AJ Woo, LJ Alvarez, CM Zicovich-Wilson, MA Camblor. In Situ Disorder-Order Transformation in Synthetic Gallosilicate Zeolites with the NAT Topology. J. Am. Chem. Soc. 
2004, 126 (42), 13742-13751. https://doi.org/10.1021/ ja046921h.

89. G Ghigo, A Maranzana, G Tonachini, CM ZicovichWilson, MA Camblor. Modeling Soot and Its Functionalization under Atmospheric or Combustion Conditions by Density Functional Theory within Molecular (Polycyclic-Aromatic-Hydrocarbon-like) and Periodic Methodologies. J. Phys. Chem. B 2004, 108 (10), 3215-3223. https://doi.org/10.1021/jp037 $011+$.

90. A Damin, FX Llabrés i Xamena, C Lamberti, B Civalleri, CM Zicovich-Wilson, A Zecchina. Structural, Electronic, and Vibrational Properties of the Ti-OTi Quantum Wires in the Titanosilicate ETS-10. $J$. Phys. Chem. B 2004, 108 (4), 1328-1336. https://doi. org/10.1021/jp036902e.

91. X Solans-Monfort, V Branchadell, M Sodupe, CM Zicovich-Wilson, E Gribov, G Spoto, C Busco, P. Ugliengo. $\mathrm{Can} \mathrm{Cu}^{+}$-Exchanged Zeolites Store Molecular Hydrogen? An Ab-Initio Periodic Study Compared with Low-Temperature. J. Phys. Chem. B 2004, 108 (24), 8278-8286. https://doi.org/10.1021/jp0486651.

92. F Pascale, S Tosoni, CM Zicovich-Wilson, P Ugliengo, R Orlando, R Dovesi. Vibrational Spectrum of Brucite, $\mathrm{Mg}(\mathrm{OH}) 2$ : A Periodic Ab Initio Quantum Mechanical Calculation Including $\mathrm{OH}$ Anharmonicity. Chem. Phys. Lett. 2004, 396 (4-6), 308-315. https://doi. org/10.1016/j.cplett.2004.08.047.

93. M Prencipe, F Pascale, CM Zicovich-Wilson, VR Saunders, R Orlando, R Dovesi. The Vibrational Spectrum of Calcite (CaCO3): An Ab Initio Quantum-Mechanical Calculation. Phys. Chem. Miner. 2004, 31 (8), 559-564. https://doi.org/10.1007/s0026 9-004-0418-7.

94. CM Zicovich-Wilson, F Pascale, C Roetti, VR Saunders, R Orlando, R Dovesi. Calculation of the Vibration Frequencies of A-quartz: The Effect of Hamiltonian and Basis Set. J. Comput. Chem. 2004, 25 (15), 1873-1881. https://doi.org/10.1002/jcc.20120.

95. F Pascale, CM Zicovich-Wilson, FL Gejo, B Civalleri, R Orlando, R Dovesi. The Calculation of the Vibrational Frequencies of Crystalline Compounds and Its Implementation in the CRYSTAL Code. J. Comput. Chem. 2004, 25 (6), 888-897. https://doi.org/10.1002/ jcc.20019.

96. RJ Corrêa, EF Sousa-Aguiar, A Ramírez-Solís, CM Zicovich-Wilson, CJ Mota. DFT Cluster Calculations for Alkali Cation-Exchanged Zeolites Interacting with Ethylchloride and HCl. J. Phys. Chem. B 2004, 108 (30), 10658-10662. https://doi.org/10.1021/jp037353f.

97. A Bert, M Llunell, R Dovesi, CM Zicovich-Wilson. Electronic Structure Characterization of Six Semiconductors through Their Localized Wannier Functions.
Phys. Chem. Chem. Phys. 2003, 5 (23), 5319-5325. https://doi.org/10.1039/b307731d.

98. CM Zicovich-Wilson. Review: Understanding the Catalytic Properties of Zeolites by Means of Quantum Chemical Tools. ChemInform 2003, 34 (27). https:// doi.org/10.1002/chin.200327290.

99. LA Villaescusa, FM Márquez, CM Zicovich-Wilson, MA Camblor. Infrared Investigation of Fluoride Occluded in Double Four-Member Rings in Zeolites. J. Phys. Chem. B 2002, 106 (10), 2796-2800. https:// doi.org/10.1021/jp013190o.

100. CM Zicovich-Wilson, A Bert, C Roetti, R Dovesi, VR Saunders. Characterization of the Electronic Structure of Crystalline Compounds through Their Localized Wannier Functions. J. Chem. Phys. 2002, 116 (3), 1120-1127. https://doi.org/10.1063/1.1425406.

101. T Blasco, A Corma, MJ Díaz-Cabañas, F Rey, JA Vidal-Moya, CM Zicovich-Wilson. Preferential Location of Ge in the Double Four-Membered Ring Units of ITQ-7 Zeolite. J. Phys. Chem. B 2002, 106 (10), 2634-2642. https://doi.org/10.1021/jp013302b.

102. F Márquez, CM Zicovich-Wilson, A Corma, E Palomares, H García. Naphthalene Included within AllSilica Zeolites: Influence of the Host on the Naphthalene Photophysics. J. Phys. Chem. B 2001, 105 (41), 9973-9979. https://doi.org/10.1021/jp012095c.

103. Ph Baranek, CM Zicovich-Wilson, C Roetti, R Orlando, R Dovesi. Well Localized Crystalline Orbitals Obtained from Bloch Functions: The Case of KNbO 3. Phys. Rev. B 2001, 64 (12), 125102. https:// doi.org/10.1103/physrevb.64.125102.

104. M Boronat, CM Zicovich-Wilson, P Viruela, MA Camblor. Cluster and Periodic Calculations of the Ethene Protonation Reaction Catalyzed by theta-1 Zeolite: Influence of Method, Model Size, and Structural Constraints. Chem. Eur. J. 2001, 7 (6), 12951303. https://doi.org/10.1002/1521-3765(20010 316)7:6<1295::AID-CHEM1295>3.0.CO;2-S.

105. M Boronat, CM Zicovich-Wilson, P Viruela, A Camblor. Influence of the Local Geometry of Zeolite Active Sites and Olefin Size on the Stability of Alkoxide Intermediates. J. Phys. Chem. B 2001, 105 (45), 11169-11177. https://doi.org/10.1021/jp011481r.

106. Y Noel, C.M Zicovich-Wilson, B Civalleri, Ph D'Arco, $\mathrm{R}$ Dovesi. Polarization Properties of $\mathrm{ZnO}$ and $\mathrm{BeO}$ : $\mathrm{An}$ Ab Initio Study through the Berry Phase and Wannier Functions Approaches. Phys. Rev. B 2001, 65 (1), 014111. https://doi.org/10.1103/physrevb.65.014111.

107. CM Zicovich-Wilson, R Dovesi, VR Saunders. A General Method to Obtain Well Localized Wannier Functions for Composite Energy Bands in Linear Combination of Atomic Orbital Periodic Calculations. $J$. 
Chem. Phys. 2001, 115 (21), 9708-9719. https://doi. org/10.1063/1.1415745.

108. P Ugliengo, B Civalleri, CM Zicovich-Wilson, R Dovesi. H-Chabazite with Variable Si/Al Ratio: Stability and $\mathrm{OH}$ Vibrational Frequency Computed in a Periodic LCAO B3LYP Approach. Chem. Phys. Lett. 2000, 318 (1-3), 247-255. https://doi.org/10.1016/ s0009-2614(00)00018-x.

109. CM Zicovich-Wilson, MA Camblor. Modifying the Catalytic Activity of Ti-Zeolites by Isomorphic Substitution of Si by Ge Atoms. A Periodic QuantumChemical Study. J. Phys. Chem. B 2000, 104 (17), 4134-4140. https://doi.org/10.1021/jp993585w.

110. E Morales, CM Zicovich-Wilson. E. Sánchez, LJ Alvarez. Charge Distribution in NaY Zeolite from ChargeTransfer Molecular Dynamics. Chem. Phys. Lett. 2000, 327 (3-4), 224-229. https://doi.org/10.1016/s0009 -2614(00)00790-9.

111. CM Zicovich-Wilson, R Dovesi, A. Camblor. Interaction of Ti-Zeolites with Water. A Periodic Ab-Initio Study. J. Phys. Chem. B 1999, 103 (6), 988-994 https ://doi.org/10.1021/jp982916m.

112. P Ugliengo, B Civalleri, R Dovesi, CM Zicovich-Wilson. Periodic B3LYP Calculations on H-Edingtonites, Both Alone and Interacting with Acetylene. Phys. Chem. Chem. Phys. 1999, 1 (4), 545-553. https://doi. org/10.1039/a807534d.

113. CM Zicovich-Wilson. Cluster and Periodic Ab Initio Study of the Ethane-Ethene Hydride Transfer Reaction Catalyzed by Acid Chabazite. Is the Cluster Model Able to Describe Accurately the Host-guest Interactions? Phys. Chem. Chem. Phys. 1999, 1 (4), 537-543. https://doi.org/10.1039/a808037b.

114. J Fernández-Sanz, CM Zicovich-Wilson. A Periodic Hartree-Fock Study of Na Adsorption on the TiO2 (110) Rutile Surface. Chem. Phys. Lett. 1999, 303 (1), 111-116. https://doi.org/10.1016/s0009 -2614(99)00195-5.

115. CM Zicovich-Wilson, R Dovesi. On the Use of Symmetry-Adapted Crystalline Orbitals in SCF-LCAO Periodic Calculations. I. The Construction of the Symmetrized Orbitals. Int. J. Quantum Chem. 1998, 67 (5), 299-309. https://doi.org/10.1002/(SICI)1097461X(1998)67:5<299::AID-QUA3>3.0.CO;2-Q.

116. CM Zicovich-Wilson, R. Dovesi. On the Use of Symmetry-Adapted Crystalline Orbitals in SCF-LCAO Periodic Calculations. II. Implementation of the Self-Consistent-Field Scheme and Examples. Int. J. Quantum Chem. 1998, 67 (5), 311-320. https://doi. org/10.1002/(sici)1097-461x(1998)67:53.0.co;2-y.

117. A Lichanot, C Larrieu, CM Zicovich-Wilson, C Roetti, R Orlando, R Dovesi. Trapped-Hole Centres Containing Lithium and Sodium in $\mathrm{MgO}, \mathrm{CaO}$ and $\mathrm{SrO}$. An
Ab Initio Supercell Study. J. Phys. Chem. Solids 1998, 59 (6-7), 1119-1124. https://doi.org/10.1016/s0022 -3697(97)00249-7.

118. CM Zicovich-Wilson, R Dovesi. Titanium-Containing Zeolites. A Periodic Ab Initio Hartree-Fock Characterization. J. Phys. Chem. B 1998, 102 (8), 1411-1417. https://doi.org/10.1021/jp972343y.

119. B Civalleri, CM Zicovich-Wilson, P Ugliengo, VR Saunders, R Dovesi. A Periodic Ab Initio Study of the Structure and Relative Stability of Silica Polymorphs. Chem. Phys. Lett. 1998, 292 (4-6), 394-402. https:// doi.org/10.1016/s0009-2614(98)00749-0.

120. CM Zicovich-Wilson, R Dovesi. On the Structure and Stability of Ti-Zeolites. A Comparison of Cluster and Periodicab Initio Calculations. Nuovo Cim. D 1997, 19 (11), 1785-1790. https://doi.org/10.1007/bf03185375.

121. CM Zicovich-Wilson, R Dovesi. Periodic Ab Initio Study of Silico-Faujasite. Chem. Phys. Lett. 1997, 277 (1-3), 227-233. https://doi.org/10.1016/s0009 -2614(97)00888-9.

122. CM Zicovich-Wilson, R Dovesi. Periodic Ab Initio Study of the Oxidizing Sites in Ti-Containing Zeolites. J. Mol. Catal. A Chem. 1997, 119 (1-3), 449-458. https://doi.org/10.1016/s1381-1169(96)00508-0.

123. F Rajadell, J Planelles, W Jaskólski, CM ZicovichWilson. Selection of Basis Sets for Atoms and Molecules in Cavities. Int. J. Quantum Chem. 1996, 60 (5), 993-999. https://doi.org/10.1002/(SICI)1097461X(1996)60:5<993::AID-QUA5>3.0.CO;2-S.

124. J Planelles, CM Zicovich-Wilson, W Jaskolski, MA. Camblor. Semiempirical Hamiltonians for Spatially Confined П-electron Systems. Int. J. Quantum Chem. 1996, 60 (5), 971-981. https://doi. org/10.1002/(SICI)1097-461X(1996)60:5<971::AIDQUA3>3.0.CO;2-U.

125. CM Zicovich-Wilson, W Jaskólski, J. Planelles. Atoms and Molecules in Cavities: A Method for Study of Spatial Confinement Effects. Int. J. Quantum Chem. 1995, 54 (1), 61-72. https://doi.org/10.1002/qua.560540109.

126. W Kolodziejski, CM Zicovich-Wilson, C Corell, J Perez-Pariente, A. Camblor. 27Al and 29Si MAS NMR Study of Zeolite MCM-22. J. Phys. Chem. 1995, 99 (18), 7002-7008. https://doi.org/10.1021/j100018a03 7.

127. CM Zicovich-Wilson, P Viruela, A. Camblor. Formation of Surface Methoxy Groups on H-Zeolites from Methanol. A Quantum Chemical Study. J. Phys. Chem. 1995, 99 (35), 13224-13231. https://doi.org/10.1021/ j100035a029.

128. CM Zicovich-Wilson, JH Planelles, W. J. Spatially Confined Simple Quantum Mechanical Systems. Int. J. Quantum Chem. 1994, 50 (6), 429-444. https://doi. org/10.1002/qua.560500606. 
129. A Corma, CM Zicovich-Wilson, P. Viruela. OrbitalControlled Reactions Catalysed by Zeolites: Electrophilic Alkylation of Aromatics. J. Phys. Org. Chem. 1994, 7 (7), 364-370. https://doi.org/10.1002/ poc. 610070706 .

130. A Corma, F Llopis, P Viruela, CM Zicovich-Wilson. Acid Softness and Hardness in Large-Pore Zeolites as a Determinant Parameter to Control Selectivity in Orbital-Controlled Reactions. J. Am. Chem. Soc. 1994, 116 (1), 134-142. https://doi.org/10.1021/ja00080a01 6.

131. CM Zicovich-Wilson, A Corma, P Viruela. Electronic Confinement of Molecules in Microscopic Pores. A New Concept Which Contributes to the Explanation of the Catalytic Activity of Zeolites. J. Phys. Chem. 1994, 98 (42), 10863-10870. https://doi.org/10.1021/ j100093a030.

132. J Planelles, CM Zicovich-Wilson. A Simple Proof for the Formula to Get Symmetrized Powers of Group Representations. Int. J. Quantum Chem. 1993, 47 (4), 319-323. https://doi.org/10.1002/qua.560470405.

133. P Viruela-Martin, CM Zicovich-Wilson, A. Camblor. $\mathrm{Ab}$ Initio Molecular Orbital Calculations of the Proto- nation of Propylene and Isobutene by Acidic Hydroxyl Groups of Isomorphously Substituted Zeolites. $J$. Phys. Chem. 1993, 97 (51), 13713-13719. https://doi. org/10.1021/j100153a047.

134. A Corma, G Sastre, R Viruela, CM Zicovich-Wilson. Molecular Orbital Calculation of the Soft-Hard Acidity of Zeolites and Its Catalytic Implications. J. Catal. 1992, 136 (2), 521-530. https://doi.org/10.1016/00219517(92)90082-s.

135. PM Viruela-Martin, R Viruela-Martin, CM ZicovichWilson, F. T.-V. Theoretical EHT Study of Oxidative Coupling of Methane on Pure $\mathrm{MgO}$ and $\mathrm{MgO}$ Doped with Li and Na. J. Mol. Catal. 1991, 64 (2), 191-200. https://doi.org/10.1016/0304-5102(91)85111-e.

136. CM Zicovich-Wilson, R González-Luque, P. Viruela. Oxidative Coupling of Methane on Mgo. Ab Initio UHF Potential Energy Surface. J. Mol. Struct. THEOCHEM 1990, 208 (3-4), 153-162. https://doi. org/10.1016/0166-1280(90)80001-5. 